\title{
Expression of HLA-DR antigen in different histological types of gastric polyp
}

\author{
A Wee, M Teh, G C Raju
}

\begin{abstract}
Aims: To study the expression of HLA$D R$ antigen in the different histological types of gastric polyps.

Methods: Ninety five cases of gastric polyps were histologically classified and examined for the presence of Helicobacter pylori, and for degree and type of inflammation. Further sections were stained immunohistochemically for HLA-DR antigen expression in the epithelium using a monoclonal antibody that was reactive to formalin-fixed paraffin wax embedded tissue.

Results: HLA-DR antigen was expressed in all of the inflammatory polyps studied $(20 / 20)$, and in most hyperplastic (12/16) and adenomatous $(4 / 6)$ polyps. Only a few fundic gland polyps (8/51) stained positively for HLA-DR antigen. Gastric polyps seem to have a greater tendency to express HLA-DR antigens than nonpolypoid gastric mucosa, even after considering the factors that may affect HLADR antigen expression, such as inflammation and the presence of $H$ pylori. Conclusions: Growth disturbances/polyp formation may be associated with increased HLA-DR antigen expression.
\end{abstract}

Class II HLA antigens comprise the gene products of the HLA-DR, DQ, and DP genes. These genes are located in the HLA-D region of the major histocompatibility complex (MHC) and they are important in the regulation of the immune response to $\mathrm{T}$-celldependent antigens. Unlike class I HLA antigens, class II antigens have a more restricted tissue distribution, being present mainly in cells involved in the immune response- $B$ lymphocytes, activated T lymphocytes, monocytes/macrophages and dendritic antigenpresenting cells such as Langerhans' cells. However, recent studies have shown that they can also be present in other tissues. ${ }^{12}$ In the stomach, in particular, it has been demonstrated that HLA-DR antigen is expressed in the epithelium in chronic gastritis. This expression was particularly marked when Helicobacter pylori organisms were present but was virtually absent in normal gastric epithelium. ${ }^{34}$ HLA-DR expression has also been observed in intestinal-type gastric carcinomas and is only very rarely present in the diffuse type of gastric carcinoma. ${ }^{5}$
Gastric polyps provide a useful model to study HLA-DR expression since the various types of polyps arise through diverse mechanisms and constitute different types of growth disorders. ${ }^{6}$ HLA-DR antigen expression of gastric polyps and correspondingly inflamed non-polypoid gastric mucosa when $H$ pylori was not present were compared to see whether these growth disorders, of themselves, are able to affect its expression.

\section{Methods}

From January 1986 to December 1991, there were 122 gastric biopsies coded under "polyps" in the department files. They were taken from patients who had undergone gastroduodenoscopy for gastrointestinal symptoms and/or surgical resection of polyps. Patients with gastric carcinoma, submucosal lesions and postgastrectomy polypoidal lesions were excluded. A final total of 95 biopsies of gastric mucosal polyps was studied after excluding insufficient material/unsuitable cases $(n=27)$. The specimens were fixed in $10 \%$ buffered formalin. Paraffin wax embedded histological sections $4 \mu \mathrm{m}$ thick were stained with haematoxylin and eosin and by the periodic acid-Schiff technique.

\section{HISTOLOGICAL ASSESSMENT}

Typing of gastric mucosal polyps

Gastric mucosal polyps were classified according to Appleman ${ }^{6}$ and Laxen et al ${ }^{7}$ with slight modifications.

Fundic gland polyp: short pits, masses of body-type glands close to the surface, and deep cystically dilated glands and/or pits.

Inflammatory polyp: inflammation only with or without loss of normal gastric glands.

Hyperplastic polyp: disorganised tubular or cystic overgrowth of hyperplastic but otherwise normal foveolar epithelium.

Focal foveolar hyperplasia: distinctly elongated, hyperplastic but structurally well organised normal gastric pits; this category was not differentiated from hyperplastic polyps.

Adenoma: abnormal tubular or villous structures lined by immature and poorly differentiated intestinal-type epithelium.

Peutz-Jeghers polyp: redundant, branching and even cystic pits with few glands; branching of the muscularis mucosae may be minimal. The lamina propria is characteristically sparse and not inflamed.

A patient was counted only once if he had more than one specimen from a single or 
Table $1 \quad H L A-D R$ antigen expression in gastric polyps

\begin{tabular}{lrrrrr}
\hline & \multicolumn{3}{c}{$H L A-D R$ antigen } & \\
\cline { 2 - 5 } Histological type & 0 & $1+$ & $2+$ & $3+$ & No of polyps \\
\hline Fundic gland & 43 & 8 & 0 & 0 & 51 \\
Inflammatory & 0 & 7 & 5 & 8 & 20 \\
Hyperplastic & 4 & 6 & 6 & 0 & 16 \\
Adenomatous & 2 & 1 & 3 & 0 & 6 \\
Peutz-Jeghers & 1 & 0 & 1 & 0 & 2 \\
Total & 50 & 22 & 15 & 8 & 95
\end{tabular}

0 , No definite staining; $1+,<10 \%$ staining; $2+, 10-50 \%$ staining; $3+,>50 \%$ staining.

multiple polyp/s of the same histological type, taken either during one procedure or on separate occasions. If a patient had histologically different polyps detected on a single or separate occasion/s, he was entered as many times as the different categories of polyps were encountered.

\section{Assessment of inflammation and $H$ pylori}

Histological gastritis was defined by a modification $^{8}$ of the system proposed by McNulty et al. ${ }^{9}$ Mononuclear and polymorphonuclear cellular infiltration were each graded from zero to four. Gastritis was diagnosed when the acute inflammatory score was one or more, or when the chronic inflammatory score was three or more. The gastritis was considered acute if the polymorphonuclear cellular score was one or more with a mononuclear cellular score of under three; and chronic if the mononuclear cellular infiltrate was three or more with no acute inflammation. Acute-on-chronic gastritis was diagnosed when the acute and chronic inflammatory scores were greater than zero and two respectively.

The presence or absence of $H$ pylori was assessed by staining with haematoxylin and eosin. In our previous studies and in a blinded review by the same pathologist (AW) this was as effective as the Giemsa method. ${ }^{810}$

\section{HLA-DR ANTIGEN}

Further $4 \mu \mathrm{m}$ thick sections were stained for the presence of HLA-DR antigen with commercially available mouse monoclonal antibody (Dako, HLA-DR/alpha) at a concentration of 1 in 50 (streptavidin-biotin method). The gastric surface/foveolar and glandular epithelium were evaluated for the pattern and extent of staining using a threepoint system. This was based on the percentages of positively staining epithelial cells in their respective locations: 0 (no staining), $1+$
$(<10 \%), 2+(10-50 \%), 3+(>50 \%)$. The positive staining of the histiocytes/lymphocytes in the lamina propria provided the necessary positive control.

\section{Results}

Ninety patients were studied, 28 male and 62 female patients with an age range of $1-82$ years (mean 49 years). Thirty seven patients had more than one gastric polyp-in 32 patients they were histologically similar and in five each had two different types of polyp. A total of 95 biopsies were studied and they were classified as follows: 51 fundic gland (54\%), 20 inflammatory $(21 \%), 16$ hyperplastic $(17 \%)$, six adenomatous $(6 \%)$ and two Peutz-Jeghers (2\%) polyps. Overall, 45 polyps stained for HLA-DR antigen (table 1); 18 polyps were colonised by $H$ pylori 45 polyps were inflamed with acute-on-chronic inflammation present in 38 , just acute inflammation in six and just chronic inflammation in one polyp (table 2 ).

\section{FUNDIC GLAND POLYPS}

Eight of the 51 polyps ( $16 \%$ ) stained for HLADR antigen (table 1). The staining was only $1+$ in extent and tended to be localised to the surface/foveolar epithelium rather than glandular epithelium (fig 1). The polyps were generally non-inflamed and only one case contained $H$ pylori. None of the fundic gland polyps which expressed HLA-DR antigen was chronically inflamed or positive for $H$ pylori; however, two of them were acutely inflamed (table 2).

\section{INFLAMMATORY POLYPS}

All of the 20 inflammatory polyps $(100 \%)$ stained positively for HLA-DR antigen and staining was often extensive (table 1) (fig 2). Most of them contained $H$ pylori $(70 \%)$. Acute-on-chronic inflammation was present in $17(85 \%)$ of the polyps and acute inflammation alone in the remaining three $(15 \%)$ (table 2$)$.

\section{HYPERPLASTIC POLYPS}

Most polyps, 12 out of $16(75 \%)$, stained positively for HLA-DR antigen and the extent of staining was evenly distributed between $1+$ and $2+$ (table 1, fig 3). Only three contained organisms $(19 \%)$. Most of the polyps were inflamed: 13 showed acute-on-chronic $(81 \%)$ and one chronic inflammation only.

ADENOMATOUS POLYPS

Four out of six polyps (67\%) were positive for

Table 2 Prevalence of Helicobacter pylori and inflammation in gastric polyps

\begin{tabular}{|c|c|c|c|c|c|c|}
\hline \multirow[b]{2}{*}{ Histological type } & \multicolumn{2}{|c|}{ Helicobacter pylori } & \multicolumn{3}{|l|}{ Inflammation } & \multirow[b]{2}{*}{ No inflammation } \\
\hline & Positive & Negative & Acute-on-chronic & Acute only & Chronic only & \\
\hline $\begin{array}{l}\text { Fundic gland } \\
\text { Inflammatory } \\
\text { Hyperplastic } \\
\text { Adenomatous } \\
\text { Peutz-Jeghers }\end{array}$ & $\begin{array}{c}1(0) \\
14(14) \\
3(2) \\
0(0) \\
0(0)\end{array}$ & $\begin{array}{c}50(8) \\
6(6) \\
13(10) \\
6(4) \\
2(1)\end{array}$ & $\begin{array}{c}2(0) \\
17(17) \\
13(11) \\
5(4) \\
1(1)\end{array}$ & $\begin{array}{l}2(2) \\
3(3) \\
0(0) \\
1(0) \\
0(0)\end{array}$ & $\begin{array}{l}0(0) \\
0(0) \\
1(1) \\
0(0) \\
0(0)\end{array}$ & $\begin{array}{l}47(6) \\
0(0) \\
2(0) \\
0(0) \\
1(0)\end{array}$ \\
\hline Total & $18(16)$ & $77(29)$ & $38(33)$ & $6(5)$ & $1(1)$ & $50(6)$ \\
\hline
\end{tabular}

Numbers in parentheses indicate the number of polyps showing HLA-DR antigen expression. 


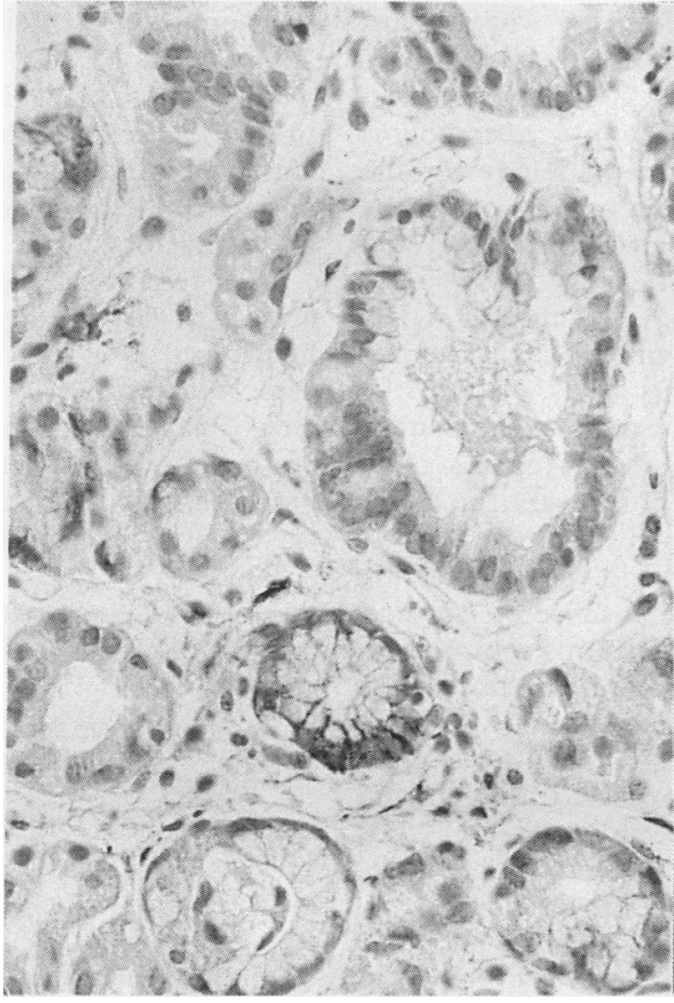

Figure 1 Immunoperoxidase staining of a fundic gland polyp showing only focal HLA-DR antigen expression in a gland.

HLA-DR antigen (table 1, fig 4). None of the polyps contained any organisms. Five $(83 \%)$ showed acute-on-chronic inflammation and the other polyp had acute inflammation only.

\section{PEUTZ-JEGHERS POLYPS}

There were only two cases of which one showed HLA-DR antigen staining. Only one polyp was inflamed with acute-on-chronic inflammation. None of the polyps contained any organisms.

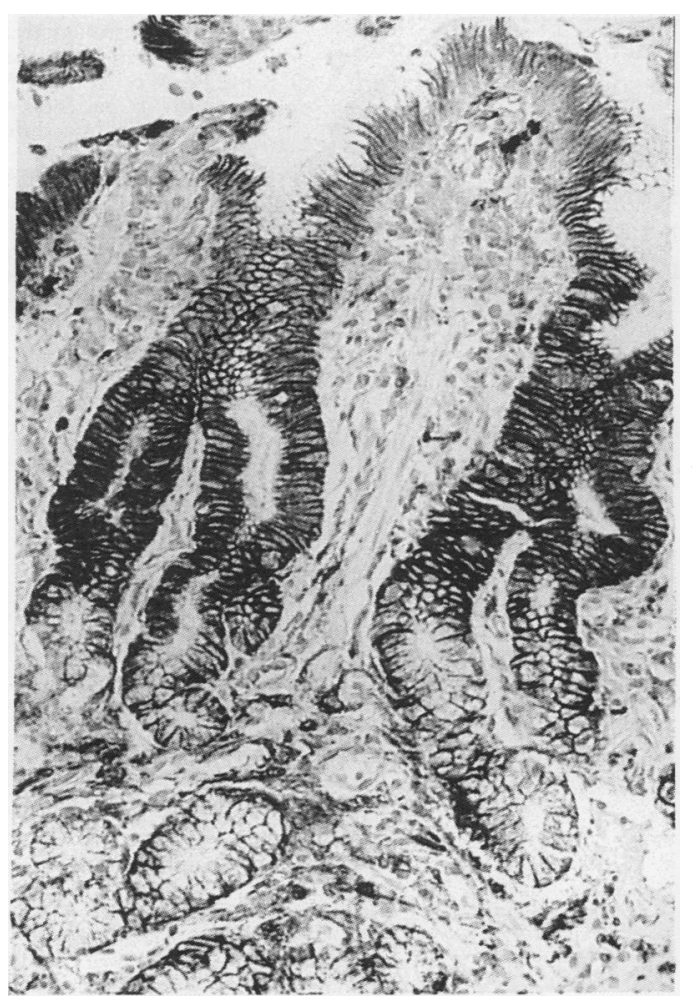

Figure 2 Immunoperoxidase staining of an inflammatory gastric polyp showing extensive $H L A-D R$ antigen expression in the epithelium.

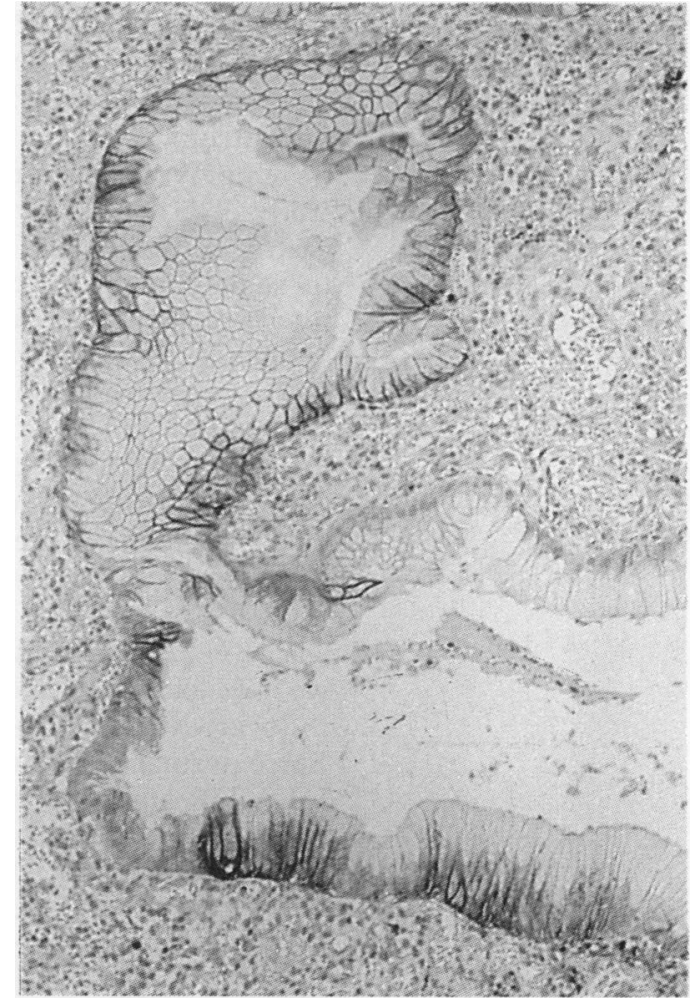

Figure 3 Immunoperoxidase staining of a hyperplastic gastric polyp showing focal $H L A-D R$ antigen expression in the hyperplastic mucus-secreting epithelium.

\section{Discussion}

We previously studied HLA-DR antigen expression in non-inflamed and inflamed gastric mucosa. In our experience, HLA-DR antigen was not expressed in histologically normal mucosa. In inflamed mucosa-acute-onchronic and chronic gastritis-the expression of this antigen was low in the antrum, being present in only seven of 18 cases $(39 \%)$ and lower still in the body mucosa when $H$ pylori

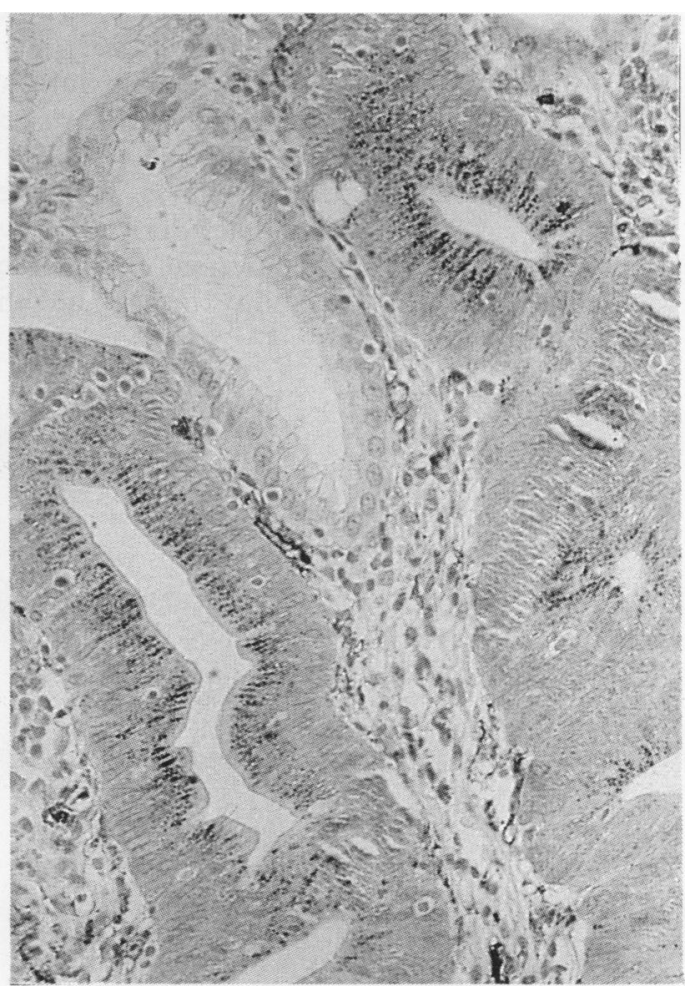

Figure 4 Immunoperoxidase staining of an adenoma showing $H L A-D R$ antigen expression in neoplastic epithelium. 
was not present. When $H$ pylori was present, the expression of the antigen was markedly increased, rising to $92 \%$ or 11 out of 12 cases in the antrum. ${ }^{3}$ For this study on gastric polyps we employed the same methodology for the study of HLA-DR antigen expression in the gastric epithelium. When we considered only those polyps which were negative for $H$ pylori, the percentage of cases showing HLA-DR antigen expression was unexpectedly high$100 \%$ of the inflammatory $(6 / 6), 77 \%$ of the hyperplastic (10/13), $67 \%$ of the adenomatous $(4 / 6)$ and $50 \%$ of the Peutz-Jeghers (1/2) polyps stained positively for the antigen (table 2). Overall, the percentage of gastric polyps, excluding fundic gland polyps, expressing HLA-DR antigen in the absence of $H$ pylori was $78 \%(21 / 27)$ (table 2). In this comparison, we have excluded fundic gland polyps because the overwhelming majority were non-inflamed. Since normal gastric mucosa does not express HLA-DR antigen, ${ }^{8}$ we expected fundic gland polyps to be similarly negative for HLA-DR antigen expression. Despite this, eight fundic gland polyps expressed HLA-DR antigen although six of these were not inflamed and none contained $H$ pylori (table 2 ).

Our results would seem to indicate that aside from inflammation and the presence of $H$ pylori, gastric polyp formation may be associated with aberrant HLA-DR antigen expression. It was not unexpected for adenomas to have a high rate of HLA-DR expression (67\%) since previous studies showed the antigen to be expressed in $74 \%$ of intestinal-type gastric carcinomas. $^{5}$ It was surprising that the prevalence of antigen expression was higher than expected, considering the inflammatory status and the presence of organisms, even for inflammatory, hyperplastic, and fundic gland polyps. One possible explanation is that all gastric mucosal polyps, neoplastic and nonneoplastic in nature, are in some way associated with the release of growth factors that may in turn be able to affect the expression of class II HLA genes. It is interesting to note that both platelet-derived growth factor (PDGF) and epidermal growth factor (EGF) may have the ability to regulate the production of gamma interferon, a potent inducer of class II HLA antigens, by $\mathrm{T}$ cells ${ }^{11}$ and increase the expression of class II MHC antigens on antigenpresenting cells. ${ }^{12}$ The increase in HLA-DR antigen expression in the epithelium of gastric polyps could be affected by these or similar growth factors.

The functional significance of HLA-DR antigen in gastric polyps is not known. Since T helper cells are able to recognise antigens in association with class II antigens, the expression of class II antigens on the surface of gastric epithelial cells may lead to the development of gastric antibodies. Further studies are necessary to test this hypothesis.

The authors are grateful to $\mathrm{Mr} \mathrm{C} \mathrm{K} \mathrm{Ow}$ for technical work, $\mathrm{Mr}$ T C Tan for photography, and Mrs A T Mok for stenographic assistance.

1 Bodmer WF. The HLA system: structure and function $J$ Clin Pathol 1987;40:948-58.

2 Klareskog L, Forsum U. Tissue distribution of class II transplantation antigens: presence on normal cells. In: Solheim BG, Moller E, Ferrone S, eds. HLA class antigens. A comprehensive review of structure and function. Berlin, Heidelberg: Springer-Verlag, 1986:339-55.

3 Wee A, Teh M, Kang JY. Association of Helicobacter pylori with HLA-DR antigen expression in gastritis. $J$ Clin Pathol 1992;45:30-3.

4 Valnes K, Huitfeldt HS, Brandtzaeg P. Relation between T cell number and epithelial HLA class II expression quantified by image analysis in normal and inflamed human gastric mucosa. Gut 1990;31:647-52.

5 Teh M, Lee YS. HLA-DR antigen expression in intestinaltype and diffuse-type gastric carcinoma. Cancer 1992 (in press).

6 Appleman HD. Localized and extensive expansions of the gastric mucosa: mucosal polyps and giant folds. In: Appleman HD, ed. Pathology of the esophagus, stomach and duodenum. Contemporary issues in surgical pathology, vol 4 New York: Churchill Livingstone, 1984:79-119.

7 Laxen F, Sipponen P, Ihamaki T, Hakkiluoto A, Dortscheva Z. Gastric polyps; their morphological and endoscopical
. characteristics and relation to gastric carcinoma. Acto Pathol Microbiol Immunol Scand 1982;90:221-8.

8 Wee A, Kang JY, Ho MS, Choong HL, Wu AYT, Sutherland IH. Gastroduodenal mucosa in uraemia: endoscopic and histological correlation and prevalence of helicobacter-like organisms. Gut 1990;10:1093-6.

9 McNulty CAM, Gearty JC, Crump B, et al. Campylobacter pyloridis and associated gastritis: investigator blind placebo controlled trial of bismuth salicylate and erythromycin ethylsuccinate. Br Med J 1986;293:645-9.

10 Kang JY, Wee A, Math MV, et al. Helicobacter pylori and gastritis in patients with peptic ulcer and non-ulcer gastritis in patients with peptic ulcer and non-ulcer
dyspepsia: ethnic differences in Singapore. Gut 1990; dyspepsia:

11 Johnson HM, Torres BA. Peptide growth factors PDGF $\mathrm{EGF}$, and FGF regulate immune interferon production $J$ Immunol 1985;134:2824-6.

12 Acres RB, Lam JR, Feldman M. Effects of PDGF and EGF on antigen-induced proliferation of human $\mathrm{T}$-cell lines. Immunology 1985;54:9-16. 